\title{
Ethnologies
}

\section{L’Union européenne et la lutte contre le trafic illicite de biens culturels}

\section{Sabrina Urbinati}

Volume 39, numéro 1, 2017

Géopolitique, conflits et patrimoine

Geopolitics, conflicts and heritage

URI : https://id.erudit.org/iderudit/1051062ar

DOI : https://doi.org/10.7202/1051062ar

Aller au sommaire du numéro

\section{Éditeur(s)}

Association Canadienne d'Ethnologie et de Folklore

ISSN

1481-5974 (imprimé)

1708-0401 (numérique)

Découvrir la revue

Citer cet article

Urbinati, S. (2017). L'Union européenne et la lutte contre le trafic illicite de biens culturels. Ethnologies, 39(1), 237-250. https://doi.org/10.7202/1051062ar
Résumé de l'article

À l'heure actuelle, nous sommes confrontés aux destructions haineuses et au trafic illicite de biens culturels en situation de conflit, comme en Iraq ou en Syrie. Outre les États, plusieurs organisations internationales sont intervenues pour contrer ces phénomènes : l'UNESCO, l'ONU, la CPI, Interpol et l'Union européenne. Même si l'on peut penser, à première vue, que cette question du trafic de biens culturels qui se pose à l'échelle internationale dépasse la compétence de l'Union européenne, il faut convenir que celle-ci est en train de jeter les bases politiques et juridiques nécessaires pour lui permettre de jouer un rôle important dans ce combat. Cet article a pour finalité de montrer ces éléments et, pour ce faire, l'auteur, tout d'abord, illustre les initiatives institutionnelles et de politique commune prises par l'Union européenne, et deuxièmement examine le cadre juridique pertinent dans ce cas. Elle consacre une attention particulière aux principaux éléments du projet de Règlement sur l'importation de biens culturels dans l’Union. 


\title{
LUUNION EUROPÉENNE ET LA LUTTE CONTRE LE TRAFIC ILLICITE DE BIENS CULTURELS
}

\author{
Sabrina Urbinati \\ Université de Milano-Bicocca
}

\section{Introduction}

Le monde est de plus en plus confronté aux destructions et endommagements haineux ainsi qu'au trafic illicite de biens culturels, en particulier dans des situations de conflit comme en Iraq et en Syrie. Depuis le début des conflits armés dans ces deux pays, le trafic illicite de leurs biens culturels s'est développé très rapidement, pour trois raisons principales : 1) les groupes terroristes de Daech et al-Qaïda détruisent les patrimoines culturels qui, bien qu'appartenant à des civilisations qui ont fait partie de l'histoire de l'Iraq et de la Syrie, donnent une vision de la religion différente de la leur, et ils utilisent les revenus dérivant du trafic illicite de biens culturels pour financer leurs activités terroristes ; 2) le pillage et la contrebande des biens culturels mis en œuvre par le crime organisé local et international, phénomènes déjà bien présents dans ces deux pays, ont augmenté exponentiellement à la faveur du chaos provoqué par les affrontements ; 3 ) enfin, il se peut que les migrants d'Iraq et de Syrie, en fuyant les conflits armés, emportent avec eux des biens culturels, des objets petits et faciles à transporter, afin de financer leur voyage. Pour faire face à ces phénomènes, à côté des interventions mises en œuvre par les États ${ }^{1}$ qui se portent à la défense de ces patrimoines culturels, on observe la montée en puissance d'organisations internationales et régionales telles que l'Organisation des Nations Unies pour l'éducation, la science et la culture (UNESCO) $)^{2}$, l'Organisation des Nations Unies (ONU), la Cour pénale

1. Par exemple, l'Italie a établi la Task Force «Unite4Heritage ».

2. Voir l'adoption de la Stratégie pour le renforcement de l'action de l'UNESCO en matière de protection de la culture et de promotion du pluralisme culturel en cas de conflit armé et, dans ce cadre, l'initiative Unite4Heritage de l'UNESCO, en ligne, http://www. unite4heritage.org/en/unite4heritage-celebrating-safeguarding-cultural-heritage ; 
internationale (CPI) ${ }^{3}$, Interpol et l'Union européenne (UE).

Bien que l'UE se présente d'abord comme l'exemple d'une intégration régionale essentiellement vouée à l'intégration économique de ses États membres, elle a désormais pleinement démontré que son ambition était politique et se situait à l'échelle internationale. De récentes initiatives institutionnelles et de politique commune prises dans le domaine de la lutte contre le trafic illicite de biens culturels constituent une illustration de ce phénomène.

Notre analyse vise d'une part à rappeler les instruments juridiques dédiés en général à la circulation des biens culturels dans le territoire de l'Union, et d'autre part à examiner ceux qui concernent directement la lutte contre le trafic en question afin de repérer les évolutions récentes.

\section{Les instruments juridiques dédiés en général à la circulation des biens culturels}

Depuis 2009, l'Union européenne a adopté plusieurs initiatives institutionnelles et politiques qui méritent d'être rappelées. Premièrement, le Traité de Lisbonne, adopté en 2009, a établi le Service européen pour l'Action extérieure, qui, depuis lors, supporte le haut-représentant de l'Union pour les affaires étrangères et la politique de sécurité. Celui-ci s'est vu confier davantage de compétences dans le domaine de la culture. Depuis cette nouveauté, l'Union européenne a adopté plusieurs documents qui dessinent une trajectoire politique : en 2015, la Stratégie régionale de l'Union européenne pour la Syrie et l'Iraq, ainsi que la lutte contre Daech (Conseil de l'Union européenne 2015) ${ }^{4}$; le Programme européen en matière de sécurité (Commission européenne 2015) ; la Résolution du Parlement européen du 30 avril 2015 sur la destruction de sites culturels

voir également les autres initiatives de l'UNESCO et d'autres sujets, en ligne, https://en.unesco.org/The-role-of-culture-for-resilience-peace-and-security.

3. Voir les deux jugements dans l'affaire al-Mahdi.

4. «L'U[union européenne] condamne fermement la destruction délibérée par l'EIIL/ Daech du patrimoine archéologique et culturel en Syrie et en Iraq et l'idéologie extrémiste qui la sous-tend, et note que de tels actes peuvent constituer des crimes de guerre en vertu du Statut de Rome de la Cour pénale internationale. L'U[union européenne] est déterminée à contribuer à documenter et protéger le patrimoine de la Syrie et de l'Iraq tout en prenant des mesures appropriées, conformément à la résolution 2199 du Conseil de sécurité des Nations unies, pour empêcher le commerce illégal de biens culturels, et fait observer que ce trafic illicite d'objets culturels contribue directement au financement de l'EIIL/Daech et d'autres organisations terroristes ». 
par le groupe État islamique (Parlement européen 2015) ; en 2016, le Plan d'action destiné à renforcer la lutte contre le financement du terrorisme (Commission européenne 2016b) ${ }^{5}$ et la Stratégie de l'Union européenne dans le domaine des relations culturelles internationales (Commission européenne 2016a). Une attention particulière est portée à la question du trafic illicite des biens culturels, non seulement parce qu'il représente une menace contre les patrimoines culturels d'Iraq et de Syrie, mais surtout parce qu'il est considéré comme une source importante de revenus pour les groupes de terroristes impliqués.

Dans le domaine de la culture, l'article 167 du Traité sur le fonctionnement de l'Union européenne attribue à l'Union une compétence supplémentaire par rapport à celles de ses États membres :

1) l'Union contribue à l'épanouissement des cultures des États membres dans le respect de leur diversité nationale et régionale, tout en mettant en évidence l'héritage culturel commun ;

2) l'action de l'Union vise à encourager la coopération entre États membres et, si nécessaire, à appuyer et compléter leur action dans les domaines suivants :

- l'amélioration de la connaissance et de la diffusion de la culture et de l'histoire des peuples européens,

- la conservation et la sauvegarde du patrimoine culturel d'importance européenne,

- les échanges culturels non commerciaux,

- la création artistique et littéraire, y compris dans le secteur de l'audiovisuel.

3) L'Union et les États membres favorisent la coopération avec les pays tiers et les organisations internationales compétentes dans le domaine

5. «Les images de la destruction de sites archéologiques par Daech, considérée par nombre de personnes comme des crimes de guerre contre le patrimoine culturel, sont fréquentes dans nos médias. Il apparaît toutefois clairement que Daech, en plus de causer des dégâts considérables, utilise les biens culturels issus des fouilles illicites de sites archéologiques comme source de revenus. Les biens culturels sortis d'Iraq et de Syrie de manière illicite peuvent constituer une source de revenus non négligeable pour les terroristes. Pour les revenus qui proviennent de produits sur les marchés européens, le fait de localiser ce commerce et d'y mettre fin pourrait avoir une incidence réelle sur une importante source de financement des activités terroristes ». 
de la culture, et en particulier avec le Conseil de l'Europe ;

4) l'Union tient compte des aspects culturels dans son action au titre d'autres dispositions des traités, afin notamment de respecter et de promouvoir la diversité de ses cultures ;

5) pour contribuer à la réalisation des objectifs visés au présent article,

- le Parlement européen et le Conseil, statuant conformément à la procédure législative ordinaire et après consultation du Comité des régions, adoptent des actions d'encouragement, à l'exclusion de toute harmonisation des dispositions législatives et règlementaires des États membres ;

- le Conseil adopte, sur proposition de la Commission, des recommandations.

L'utilisation de verbes et expressions tels que " encourager », " promouvoir », « appuyer et compléter », « contribuer à l'épanouissement » et « contribuer à la réalisation », confirme que l'Union européenne, dans le domaine en question, ne joue son rôle qu'après ses États membres et à côté d'eux.

Pour ce qui est plus spécifiquement de la circulation des biens culturels dans le territoire de l'Union, elle est soumise à la même réglementation que les marchandises dites « communes » (Titre II, Libre circulation des marchandises, articles 28 à 30 et 34 à 36 du Traité sur le fonctionnement de l'Union européenne), caractérisée par l'interdiction des droits de douane à l'importation et à l'exportation ainsi que des taxes ayant effets équivalents (articles 28 à 30 $)^{6}$ et par l'interdiction des restrictions quantitatives à

6. «Article 28-1. L'Union comprend une union douanière qui s'étend à l'ensemble des échanges de marchandises et qui comporte l'interdiction, entre les États membres, des droits de douane à l'importation et à l'exportation et de toutes taxes d'effet équivalent, ainsi que l'adoption d'un tarif douanier commun dans leurs relations avec les pays tiers. 2. Les dispositions de l'article 30 et du chapitre 3 du présent titre s'appliquent aux produits qui sont originaires des États membres, ainsi qu'aux produits en provenance de pays tiers qui se trouvent en libre pratique dans les États membres. Article 29. Sont considérés comme étant en libre pratique dans un État membre les produits en provenance de pays tiers pour lesquels les formalités d'importation ont été accomplies et les droits de douane et taxes d'effet équivalent exigibles ont été perçus dans cet État membre, et qui n'ont pas bénéficié d'une ristourne totale ou partielle de ces droits et taxes. Article 30. Les droits de douane à l'importation et à l'exportation ou taxes d'effet équivalent sont interdits entre les États membres. Cette interdiction s'applique également aux droits de douane à caractère fiscal ». 
l'exportation et à l'importation, ainsi que des mesures ayant des effets équivalents (articles 34 à 36) ${ }^{7}$.

En effet, dans le Traité sur le fonctionnement de l'Union européenne, les spécificités des biens culturels sont prises en compte par le biais de l'établissement d'une exception à l'interdiction de toute restriction à l'importation, exportation et transit de marchandises. Exception qui, cependant, a un effet limité car elle ne concerne que les biens culturels pouvant entrer dans la catégorie des " trésors nationaux ayant une valeur artistique, historique ou archéologique » (article 36 du Traité sur le fonctionnement de l'Union européenne). Ainsi, tous les biens culturels qui ne sont pas des trésors nationaux des États membres de l'Union, et donc également les biens culturels provenant de pays tiers, peuvent circuler librement dans l'Union européenne.

À côté de ces règles juridiques qui ont pour objet direct la circulation des biens culturels, il convient de rappeler qu'il y en a d'autres, à savoir celles concernant l'union douanière et la politique commerciale commune. Dans ces deux domaines, l'Union européenne a une compétence exclusive ${ }^{8}$ et c'est justement sur cette base que l'Union a pu adopter une partie des instruments sources secondaires de son droit. En effet, affirmer que l'Union européenne a une compétence exclusive dans ces domaines équivaut à dire que les États membres ont renoncé totalement à leurs pouvoirs de réglementation en faveur de l'organisation en question.

\section{Les règles juridiques de l'Union européenne pour la lutte contre le trafic illicite des biens culturels}

À côté du Traité sur le fonctionnement de l’Union européenne, il

7. «Article 34. Les restrictions quantitatives à l'importation ainsi que toutes mesures d'effet équivalent, sont interdites entre les États membres. Article 35. Les restrictions quantitatives à l'exportation, ainsi que toutes mesures d'effet équivalent, sont interdites entre les États membres. Article 36. Les dispositions des articles 34 et 35 ne font pas obstacle aux interdictions ou restrictions d'importation, d'exportation ou de transit, justifiées par des raisons de moralité publique, d'ordre public, de sécurité publique, de protection de la santé et de la vie des personnes et des animaux ou de préservation des végétaux, de protection des trésors nationaux ayant une valeur artistique, historique ou archéologique ou de protection de la propriété industrielle et commerciale. Toutefois, ces interdictions ou restrictions ne doivent constituer ni un moyen de discrimination arbitraire ni une restriction déguisée dans le commerce entre les États membres ».

8. «Article 3-1. L'Union dispose d'une compétence exclusive dans les domaines suivants : a) l'union douanière ; $[.$. . e) la politique commerciale commune $[. .$.$] ».$ 
convient de rappeler les sources de type secondaire du droit européen, soit, d'une part, le Règlement no 116/2009 du Conseil du 18 décembre 2008 concernant l'exportation de biens culturels (Conseil de l'Union européenne 2008) et la Directive 2014/60/UE du Parlement européen et du Conseil du 15 mai 2014 relative à la restitution de biens culturels ayant quitté illicitement le territoire d'un État membre ; d'autre part, le Règlement (CE) $\mathrm{n}^{\circ}$ 1210/2003 du Conseil du 7 juillet 2003 concernant certaines restrictions spécifiques applicables aux relations économiques et financières avec l'Iraq (Conseil de l'Union européenne 2003) et le Règlement (UE) n 36/2012 du Conseil du 18 janvier 2012 relatif aux mesures restrictives en raison de la situation en Syrie (Conseil de l'Union européenne 2012). À côté de ces instruments juridiques déjà existants, il est nécessaire de mentionner le projet de Règlement du Parlement européen et du Conseil concernant l'importation de biens culturels (Commission européenne 2017).

On peut distinguer deux types d'instruments déjà existants : ceux concernant la circulation et le trafic illicite de biens culturels de façon générale ; ceux prenant en considération les situations spécifiques de l'Iraq et de la Syrie.

Pour ce qui est des premiers instruments, il convient de noter que la Directive n'est applicable qu'aux restitutions de biens culturels entre États membres de l'Union européenne et qui se trouvent encore sur le territoire de l'un de ces derniers, lorsque le Règlement peut être utilisé également par rapport aux biens culturels provenant d'autres pays. En effet, le Règlement prévoit que l'exportation de biens culturels hors du territoire de l'Union est subordonnée à la présentation d'une autorisation délivrée sur demande de l'intéressé par les autorités compétentes de l'État membre sur le territoire duquel le bien culturel en question se trouve. Cette règle concerne également les biens culturels de pays tiers importés dans l'Union européenne et qui se trouvent sur le territoire d'un de ses États membres?. Ainsi, tout bien culturel d'Iraq et de Syrie importé licitement ou illicitement dans l'Union européenne pour être exporté depuis ce même territoire devra

9. «Article 2. Autorisation à l'exportation. 1. L'exportation de biens culturels hors du territoire douanier de la Communauté est subordonnée à la présentation d'une autorisation d'exportation. 2. L'autorisation d'exportation est délivrée sur demande de l'intéressé : a) par une autorité compétente de l'État membre sur le territoire duquel le bien culturel en question se trouvait, légalement et à titre définitif, au $1^{\text {er }}$ janvier 1993 ; b) ou, après cette date, par une autorité compétente de l'État membre sur le territoire duquel il se trouve après envoi légal et définitif d'un autre État membre, ou importation d'un pays tiers, ou réimportation d'un pays tiers après envoi légal d'un État membre audit pays tiers [...]». 
également être accompagné par une autorisation d'exportation.

Les deux derniers règlements ont été adoptés par l'Union européenne consécutivement, surtout, aux résolutions 1483 (2003) et 2199 (2015) du Conseil de sécurité des Nations Unies, intitulées respectivement « La situation entre l'Iraq et le Koweit » et « Menaces contre la paix et la sécurité internationales résultant d'actes de terrorisme »(ONU, Conseil de sécurité 2003 ; ONU, Conseil de sécurité 2015b ; Schmalenbach 2016 ; Scovazzi 2017b). Ils concernent spécifiquement les situations de l'Iraq et de la Syrie. Une question mériterait d'être posée : pourquoi l'Union européenne, qui n'est pas membre des Nations Unies, a-t-elle ressenti le besoin de traduire dans son ordre juridique interne les contenus de ces deux résolutions du Conseil de sécurité ? Principalement parce que ces résolutions ont été adoptées dans le cadre du Chapitre VII de la Charte des Nations Unies intitulé «Action en cas de menace contre la paix, de rupture de la paix et d'actes d'agression »; comme les États membres de l'Union européenne sont également membres de l'Organisation des Nations Unies, ils sont obligés de transposer dans leur droit interne les contenus de ces résolutions et ceci bien qu'ils puissent être contraires aux règles du marché commun européen. Ainsi, afin de garantir la cohérence du marché commun européen, l'Union européenne a-t-elle décidé d'intervenir avec ces deux règlements.

Sur la base de la lecture littérale des titres desdits règlements, il est possible de s'apercevoir que, comme les résolutions du Conseil de sécurité précédemment citées, ces instruments ont des objectifs plus généraux que celui de la protection du patrimoine culturel et de la lutte contre le trafic illicite des biens culturels en cas de conflit armé. En effet, ils concernent de façon générale les échanges commerciaux de l'Union européenne et de ses États membres avec l'Iraq et la Syrie, afin de lutter contre le financement du terrorisme. Ainsi, parmi les nombreux articles de ces deux instruments, il y en a un dans chaque règlement concernant le commerce de biens culturels de ces deux pays et la lutte contre leur trafic illicite.

L'article $3^{10}$ du Règlement concernant l'Iraq interdit l'importation ou

10. «Article 3-1. Il est interdit : a) d'importer ou d'introduire sur le territoire de la Communauté ; b) d'exporter ou de faire sortir du territoire de la Communauté ; et c) d'échanger des biens culturels iraquiens et d'autres biens présentant une importance archéologique, historique, culturelle, scientifique rare ou religieuse, y compris les biens dont la liste figure à l'annexe II, lorsqu'ils ont été sortis illégalement de sites iraquiens, et notamment lorsque i) ces biens font partie intégrante des collections publiques figurant sur les inventaires des musées, des archives et des fonds de conservation des bibliothèques iraquiens ou sur les inventaires des institutions religieuses iraquiennes, ou ii) il existe un doute raisonnable concernant le fait 
l'introduction sur le territoire de l'Union européenne, l'exportation ou la sortie de ce même territoire, ainsi que tout échange, de biens culturels iraquiens et d'autres biens présentant une importance archéologique, historique, culturelle, scientifique rare ou religieuse, lorsqu'ils sont sortis illégalement de sites iraquiens. Cet article est applicable à tous les biens culturels faisant partie des collections publiques ou pour lesquels il existe un doute raisonnable concernant le fait qu'ils sont sortis d'Iraq sans le consentement de leur propriétaire légitime ou en violation des règles juridiques iraquiennes. Ces interdictions ne concernent pas les biens culturels exportés de l'Iraq avant le 6 août 1990 ou qui ont été rendus aux institutions iraquiennes selon ce qui est établi dans le paragraphe 7 de la résolution 1483 (2003) du Conseil de sécurité ${ }^{11}$ (ONU, Conseil de sécurité 2003 ; Urbinati 2013).

\section{L'article 11-quater ${ }^{12}$ du Règlement concernant la Syrie interdit}

que ces biens ont pu être sortis d'Iraq sans le consentement de leur propriétaire légitime ou en violation des lois et de la réglementation iraquiennes. 2. Ces interdictions ne s'appliquent pas lorsqu'il est démontré que : a) ces biens culturels ont été exportés d'Iraq avant le 6 août 1990 ou b) ces biens culturels sont restitués aux institutions iraquiennes conformément à l'objectif de restitution en bon état défini au paragraphe 7 de la résolution 1483 (2003) du Conseil de sécurité des Nations Unies ».

11. «Décide que tous les États Membres doivent prendre les mesures voulues pour faciliter la restitution, en bon état, aux institutions iraquiennes des biens culturels iraquiens et des autres objets ayant une valeur archéologique, historique, culturelle, scientifique ou religieuse, qui ont été enlevés illégalement du Musée national iraquien, de la Bibliothèque nationale et d'autres sites en Iraq depuis l'adoption de la résolution 661 (1990) du 6 août 1990, notamment en frappant d'interdiction le commerce ou le transfert de ces objets et des objets dont il y a de bonnes raisons de croire qu'ils ont été enlevés illégalement et appelle l'Organisation des Nations Unies pour l'éducation, la science et la culture, Interpol et autres organisations internationales compétentes à faciliter la mise en œuvre du présent paragraphe ».

12 «Article 11-quater-1. Il est interdit d'importer, d'exporter, de transférer ou de fournir des services de courtage liés à l'importation, à l'exportation ou au transfert de biens culturels syriens et d'autres biens présentant une importance archéologique, historique, culturelle, scientifique, rare ou religieuse, y compris les biens dont la liste figure à l'annexe XI, lorsqu'il existe de bonnes raisons de soupçonner que ces biens ont été sortis de Syrie sans le consentement de leur propriétaire légitime ou ont été sortis de Syrie en violation du droit syrien ou du droit international, notamment lorsque ces biens font partie intégrante des collections publiques figurant sur les inventaires des fonds de conservation des musées syriens, des archives ou des bibliothèques, ou sur les inventaires des institutions religieuses syriennes. 2. L'interdiction énoncée au paragraphe 1 ne s'applique pas s'il est prouvé que : a) les biens ont été exportés de Syrie avant le 15 mars 2011 ; ou b) les biens sont restitués en toute sécurité à leurs propriétaires légitimes en Syrie ». 
l'importation, l'exportation et le transfert ou la fourniture des services de courtage liés à de telles activités ayant comme objet des biens culturels syriens et d'autres biens présentant une importance archéologique, historique, culturelle, scientifique, rare ou religieuse. Cet article s'applique à tous les biens pour lesquels il existe de bonnes raisons de soupçonner qu'ils ont été sortis de Syrie sans le consentement de leur propriétaire légitime ou qu'ils ont été sortis de Syrie en violation du droit de ce pays ou du droit international, notamment s'ils appartiennent à des collections publiques. Ces interdictions ne s'appliquent pas aux biens culturels exportés de Syrie avant le 15 mars 2011, ou à ceux qui ont été restitués à leur légitime propriétaire.

\section{Le projet de Règlement sur l'importation de biens culturels sur le territoire de l'Union européenne}

Le 13 juillet 2017, la Commission européenne a proposé au Conseil de l'Union européenne et au Parlement européen un projet de règlement concernant l'importation de biens culturels sur le territoire de l'Union européenne. L'adoption définitive de ce règlement est attendue en 2018.

Cette initiative de la Commission européenne se base, du point de vue juridique, sur l'article 3 du Traité sur le fonctionnement de l'Union européenne, où, ainsi que nous l'avons déjà rappelé, il est prévu que l'Union ait une compétence exclusive en matière d'union douanière et de politique commerciale commune. Du point de vue politique, cette proposition trouve son fondement dans le Programme européen en matière de sécurité (Commission européenne 2015), la Stratégie de l'Union européenne dans le domaine des relations culturelles internationales (Commission européenne 2016a) et le Plan d'action destiné à renforcer la lutte contre le financement du terrorisme (Commission européenne 2016b). Dans ce cadre, il convient également de rappeler qu'en mars 2017, le Conseil de sécurité des Nations Unies a adopté la résolution 2347, intitulée « Maintien de la paix et de la sécurité internationales », où il a demandé à ses États membres (et membres de l'Union européenne aussi) de prendre des mesures pour contrer le commerce illicite et le trafic de biens culturels, en particulier lorsqu'ils proviennent d'une situation de conflit armé, même par des groupes terroristes (Hausler 2018 ; Jakubowski 2018 ; ONU, Conseil de sécurité 2017 ; Urbinati 2018).

La proposition de règlement de la Commission vise à empêcher l'importation et le stockage dans l'Union européenne de biens culturels exportés illicitement depuis un pays tiers. Selon l'organe en question, 
ceci « permettra de réduire le trafic de biens culturels, de lutter contre le financement du terrorisme et de protéger le patrimoine culturel, en particulier les objets archéologiques provenant de pays-sources touchés par des conflits armés » (Commission européenne 2017). À cette fin, les éléments principaux de ce règlement sont : 1) l'introduction d'une définition « commune » du bien culturel ${ }^{13} ; 2$ ) l'adoption d'un système de licences à l'importation de certains types de biens culturels lorsque, pour d'autres, une déclaration signée sera suffisante ; 3) l'attribution aux services douaniers du pouvoir de saisie en cas de soupçon d'exportation illicite.

Pour ce qui est de la définition du «bien culturel », il convient de s'arrêter sur la signification de l'épithète « commune » utilisée par la Commission. En effet, il a été à maintes reprises mis en évidence que les instruments juridiques internationaux (UNESCO 1954a ; UNESCO 1954b ; UNESCO 1970 ; UNESCO 1999 ; UNIDROIT 1995) et européens existants ne se réfèrent pas à une approche définitionnelle « commune » des «biens culturels ». En outre, il est important de prendre en considération les articles du projet de règlement qui établissent que l'entrée de biens culturels sur le territoire douanier de l'Union n'est autorisée que lorsqu'une licence d'importation a été obtenue pour ceux-ci, ou qu'une déclaration de l'importateur est présentée. Les seules exceptions à ces normes concernent les importations temporaires sur le territoire de l'Union européenne liées à la recherche scientifique et universitaire ou lorsqu'il est nécessaire de protéger les biens culturels d'autres pays de la destruction et de la perte lorsque le pays-source est touché par un conflit armé ou une catastrophe naturelle. Ces articles sont fondamentaux car, jusqu'à présent, ce domaine a été laissé entièrement à l'arbitre et aux possibilités de chaque État membre et une telle situation a permis l'entrée sur le territoire et dans le marché commun de l'Union européenne de biens culturels ayant une provenance douteuse, contribuant ainsi au financement du terrorisme et du crime organisé international.

Le pouvoir de saisie confié aux services douaniers en cas de soupçon d'importation illicite est une première. Sa mise en œuvre sera à observer attentivement. En effet, un tel type de pouvoir n'est pas prévu dans le règlement concernant l'exportation des biens culturels hors du territoire de l’Union européenne, qui est un instrument juridique visant, en premier

13. «Article 2-1. Aux fins du présent règlement, on entend par : a) « bien culturels» : tout objet présentant de l'importance pour l'archéologie, la préhistoire, l'histoire, la littérature, l'art ou la science et qui appartient aux catégories énumérées dans le tableau de l'annexe et satisfait au seuil d'ancienneté minimal qui y est spécifié ». 
lieu, la protection du patrimoine culturel des États membres. Actuellement, en effet, l'utilisation du pouvoir de saisie, soit pour l'importation, soit pour l'exportation des biens culturels, est réglé par les droits internes des États membres de l'Union européenne, ce qui a pour conséquence la nonhomogénéité du contrôle aux frontières. En outre, il convient de souligner que ce pouvoir et son exercice impliquent que les bureaux de douane soient équipés de locaux adaptés à la conservation des biens culturels.

\section{Conclusion}

On assiste donc au développement de l'attention que l'Union européenne porte à la lutte contre le trafic illicite de biens culturels en temps de guerre et de paix, que ces biens appartiennent aux États-membres de l'UE ou à des pays tiers. Sur la base des analyses du cadre politico-institutionnel et juridique, il est possible d'affirmer que cette intensification est due à l'association de la lutte contre le trafic illicite de biens culturels à la lutte contre le financement du terrorisme, et non pas à une volonté de protéger le patrimoine culturel des pays tiers. Néanmoins, cela ne signifie pas que l'Union européenne ne soit pas en position de jouer un rôle important dans ce domaine ; il semble même possible d'affirmer que l'Union est en train de jeter les bases nécessaires pour jouer un rôle important dans la lutte contre ce phénomène. Cette conclusion est d'autant plus importante si l'on considère que dans les années à venir les biens culturels soustraits non seulement à l'Iraq et à la Syrie, mais également à d'autres pays en guerre, commenceront à émerger sur le marché de l'art ; l'Union européenne, en tant que marché unique mais aussi en tant qu'acteur géopolitique, doit être en mesure de faire face à ce phénomène. 


\section{Références}

Commission européenne, 2015, Le programme européen en matière de sécurité, 28 avril 2015, COM (2015) 185 final.

, 2016a, Vers une stratégie de l'UE dans le domaine des relations culturelles internationales, 8 juin 2016, JOIN (2016) 29 final.

— 2016b, Plan d'action destiné à renforcer la lutte contre le financement du terrorisme, 2 février 2016.

- 2017, Proposition de Règlement du Parlement européen et du Conseil concernant l'importation de biens culturels, 13 juillet 2017, COM (2017) 375 final.

Conseil de l'Union européenne, 2003, Règlement n. 1210/2003 concernant certaines restrictions spécifiques applicables aux relations économiques et financières avec l'Iraq, 7 juillet 2003, JO L 169.

—, 2008, Règlement n. 116/2009 du Conseil concernant l'exportation de biens culturels, 18 décembre 2008, JO L 39, 10 février 2009.

- 2012, Règlement $n .36 / 2012$ concernant des mesures restrictives en raison de la situation en Syrie, 18 janvier 2012, JO L 016, 19 janvier 2012. ,2015, Stratégie régionale de l'Union européenne pour la Syrie et l'Iraq, ainsi que pour la menace que constitue l'Eiil/Daech, 16 mars 2015.

Cour pénale internationale (CPI), 2016, Procureur c. Ahmad Al Faqi Al Mahdi, jugement ICC-01/12-01/15, 27 septembre 2016.

—, 2017, Procureur c. Ahmad Al Faqi Al Mahdi, ordonnance ICC01/12-01/15, 17 août 2017.

Frigo, Manlio, 2007, La circolazione internazionale dei beni culturali. Diritto internazionale, diritto comunitario e diritto interno. Milan, Giuffré Editore.

— 2014, "Circulation des biens culturels, détermination de la loi applicable et méthodes de règlement des litiges ». Recueil des cours de l'Académie de droit international de la Haye 375 : 1-474.

Hausler, Kristin, 2018, «Cultural Heritage and the Security Council : Why Resolution 2347 Matters ». QIL - Question of International Law. QIL, Zoom-in 48 (2018), 5-19.

Jakubowski, Andrzej, 2018, «Resolution 2347 : Mainstreaming the Protection of Cultural Heritage at the Global Level ». QIL - Question of International Law. QIL, Zoom-in 48 (2018), 21-44

ONU, Conseil de Sécurité, 2003, La situation entre l'Iraq et le Koweit. S/ RES/1483 (2003), 23 mai.

— 2015a, Menaces contre la paix et la sécurité internationales résultant d'actes de terrorisme. S/RES/2253 (2015), 17 décembre.

— 2015b, Menaces contre la paix et la sécurité internationales résultant d'actes de terrorisme. S/RES/2199 (2015), 12 février 2015. 
, 2017, Maintien de la paix et de la sécurité internationales. S/RES/2347 (2017), 24 mars 2017.

Parlement européen, 2015, Résolution du Parlement européen du 30 avril 2015 sur la destruction de sites culturels par le groupe État islamique. 30 avril 2015, JO C 346/55, 21 septembre 2016.

Parlement européen et Conseil de l'Union européenne, Directive 2014/60/ UE relative à la restitution de biens culturels ayant quitté illicitement le territoire d'un État membre. 15 mai 2014, JO L 159, 28 mai 2014.

Rossi, Pierfrancesco, 2017, « The Al Mahdi Trial Before the International Criminal Court : Attacks on Cultural Heritage between War Crimes and Crimes against Humanity ». Diritti umani e diritto internazionale : $87-100$.

Schmalenbach, Kirsten, 2016, «Ideological Warfare against Cultural Property : UN Strategies and Dilemmas ». Max Planck Yearbook of United Nations Law $20: 1-38$

Scovazzi, Tullio, 2017a, "La prima sentenza della Corte penale internazionale in tema di distruzione di beni culturali ». Diritti umani e diritto internazionale 2017 (1) : 77-86.

, « Il traffico illecito di beni culturali: non soltanto una minaccia alla pace e alla sicurezza internazionali ». Dans Ennio Triggiani et al. (dir.), Dialoghi con Ugo Villani : 197-205. Bari, Cacucci Editore.

Traité sur le fonctionnement de l'Union européenne, 26 octobre 2012, JO C 326/49.

UNESCO, 1954a, Convention pour la protection des biens culturels en cas de conflit armé. Paris, UNESCO

-, 1954b, Protocole à la Convention pour la protection des biens culturels en cas de conflit armé. Paris, UNESCO.

, 1970, Convention concernant les mesures à prendre pour interdire et empêcher l'importation, l'exportation et le transfert de propriété illicites des biens culturels. Paris, UNESCO

, 1999, Deuxième protocole relatif à la Convention de La Haye de 1954 pour la protection des biens culturels en cas de conflit armé. Paris, UNESCO.

-, 2016, Memorandum of Understanding between the Government of the Italian Republic and the United Nations Educational, Scientific and Cultural Organization on the Italian National Task Force in the Framework of UNESCO's Global Coalition Unite4Heritage for Initiatives in Favour of Countries Facing Emergencies that May Affect the Protection and Safeguarding of Culture and the Promotion of Cultural Pluralism. Rome, 16 février, en ligne : http://www.beniculturali.it/mibac/ multimedia/MiBAC/documents/1455616287505_2._Memorandum_ 
of_Understanding___11_II_2016_DRAFT_Finale_UNESCO_ versione_Italia.pdf

, 2017, Stratégie pour le renforcement de l'action de l'UNESCO en matière de protection de la culture et de promotion du pluralisme culturel en cas de conflit armé. 39C/Res 57.

UNIDROIT, 1995, Convention d'UNIDROIT sur les biens culturels volés ou illicitement exportés. Rome, UNIDROIT.

Urbinati, Sabrina, 2013, «Improving the Principle of Cooperation against Illegal Movement of Cultural Property ». TDM 5 - Transnational Dispute Management, en ligne : https://www.transnational-dispute-management. com/article.asp?key=2006

, 2018, "The Evolving Role of the United Nations Security Council and the Protection of Cultural Heritage in the Event of Armed Conflict ». QIL - Question of International Law. QIL, Zoom-in 48 (2018), 1-4. 